\title{
LIVING WELL AFTER CANCER: THE IMPACT OF SOCIAL SUPPORT AND PRODUCTIVE LEISURE
}

\author{
Cecile J. Proctor ${ }^{1}$, Danie A. Beaulieu ${ }^{1}$, Anthony J. Reiman ${ }^{2}$, \& Lisa A. Best ${ }^{1}$ \\ ${ }^{l}$ Department of Psychology, University of New Brunswick (Canada) \\ ${ }^{2}$ Department of Oncology, Saint John Regional Hospital (Canada)
}

\begin{abstract}
It is now recognized that the "cancer experience" extends beyond diagnosis, treatment, and end-of-life care. Relative to individuals who have not faced a cancer diagnosis, cancer survivors report increased mental health concerns and lowered physical and psychological well-being (Langeveld et al., 2004). Health-related quality of life encompasses overall physical (e.g., energy, fatigue, pain, etc.) and psychological functioning (e.g., emotional well-being, etc.), as well as general health perceptions (Hays \& Morales, 2001). Nayak and colleagues (2017) reported that $82.3 \%$ of cancer patients had below-average quality of life scores, with the lowest scores found in the general, physical, and psychological well-being domains. Research suggests that various positive lifestyle variables, including social connectedness, leisure activity, and mindfulness practices are associated with increased quality of life in cancer patients (Courtens et al., 1996; Fangel et al., 2013; Garland et al., 2017). In this study, 350 cancer survivors completed an online questionnaire package that included a detailed demographic questionnaire with medical and online support and leisure activity questions. Additional measures were included to assess quality of life (QLQ-C30; Aaronson et al., 1993), social connectedness (Social and Emotional Loneliness Scale for Adults, SELSA-S; DiTommaso et al., 2004), and mindfulness (Adolescent and Adult Mindfulness Scale, AAMS; Droutman et al., 2018). Results show that increased QOL is predicted by increased medical support, lower family loneliness, self-acceptance, and engaging in a variety of leisure activities. Encouraging family support, including the patient in the decision-making process, encouraging a variety of physically possible leisure activities, and normalizing negative emotions surrounding diagnosis and disease symptoms are all ways that overall QoL can be improved.
\end{abstract}

Keywords: Cancer, quality of life, leisure satisfaction, social connectedness, mindfulness.

\section{Introduction}

Globally, 1 in 6 deaths are caused by cancer, making it the second leading cause of death (World Health Organization, 2021). It is recognized that the "cancer experience" extends beyond diagnosis, treatment, and end-of-life care. Relative to individuals who have not had a cancer diagnosis, cancer survivors report increased mental health concerns and lowered physical and psychological well-being (Langeveld et al., 2004). Health-related quality of life (QoL) encompasses overall physical (e.g., energy, fatigue, pain, etc.) and psychological functioning (e.g., emotional well-being, etc.), as well as general health perceptions (Hays \& Morales, 2001). In Nayak and colleagues (2017), 82.3\% of cancer patients reported below-average QoL scores, with the lowest scores found in the general, physical, and psychological well-being domains compared to the familial, cognitive, and economic well-being domains.

Research suggests that a variety of positive lifestyle variables, including social connectedness, leisure activity, and mindfulness practices, are associated with increased QoL in cancer patients (Courtens et al., 1996; Fangel et al., 2013; Garland et al., 2017). Many studies have found that social connectedness is related to improved cancer outcomes, including decreased risk of cancer mortality and favourable prognosis (Garssen, 2004; Kroenke et al., 2006). Cancer patients often report moderate to moderately high loneliness levels, which increase with time since the initial diagnosis (Deckx et al., 2014). Further, leisure satisfaction can be described as "the positive perceptions or feelings which an individual forms, elicits, or gains as a result of engaging in leisure activities and choices" (Beard \& Ragheb, 1980, p. 22). Due to its debilitating consequences, cancer treatment and survivorship are negatively related to participation and satisfaction with leisure activities (Shipp et al., 2015). Chun and colleagues (2016) found that leisure satisfaction was a stronger predictor of an increased sense of purpose in cancer patients' life than leisure activities, lifetime trauma occurrence, and demographic factors. Moreover, dispositional mindfulness can be described as an individual's ability to "paying attention in a particular way: on purpose, in the present moment, and non-judgementally" (Kabat-Zinn, 1994, p. 4). In cancer patients, dispositional mindfulness 
has been associated with lower symptom severity for anxiety, depression, and posttraumatic stress disorder (Liu et al., 2021) and increased focus on favourable experiences, which increased overall QoL (Garland et al., 2017).

\section{Purpose of the current study}

Considering the high prevalence of cancer and its associated detrimental consequences, more research on improving cancer survivors' QoL is needed. Given that modifiable factors can have a positive impact on both physical and psychological health outcomes, the general purpose of this study was to examine how medical and social support, engagement in leisure activities, and mindfulness practices improved the QoL in cancer survivors.

\section{Method}

In total, 350 cancer survivors $\left(\mathrm{M}_{\mathrm{age}}=53.54, \mathrm{SD}=14.15\right)$ completed an online questionnaire package $\left(73.9 \%\right.$ females). The reported age of diagnosis ranged from 7-83 years $\left(\mathrm{M}_{\mathrm{age}}=46\right)$, and the years since the diagnosis ranged from 0 to 43 years $(M=6.5$ years $)$. In terms of prognosis, $34 \%$ of respondents had an initial diagnosis of cancer in stages $0-2$, while $54.5 \%$ reported that their cancer was at stages 3-4 at diagnosis (11.5\% did not know their stage). At the time of the study, $23.7 \%$ of survivors had experienced a cancer relapse, and almost all (91.1\%) of participants received some kind of treatment for their cancer since their initial diagnosis. Over half $(62.6 \%)$ of the respondents were in a romantic relationship at the time of the study.

The online questionnaire package included a demographic questionnaire with questions about medical support (e.g., Looking back on all of your treatment, how often did you feel like you could talk to your doctors (or nurses) about any concerns related to your treatment?). Leisure engagement was measured by considering the varied leisure activities of participants; more leisure activities are associated with higher leisure engagement scores. The Quality of Life of Cancer Patients Questionnaire (QLQ-C30; Aaronson et al., 1993) was used to assess cancer patients' quality of life. The QLQ-C30 includes functional subscales (Physical, Role, Cognitive, Emotional, and Social; $\alpha=.55$ to .86), three symptom subscales (Fatigue, Pain, and Nausea and Vomiting; $\alpha=.75$ to .88$)$, and a Global Health and Quality of Life subscale $(\alpha=.89)$. The 30-item questionnaire uses a 4-point Likert scale ranging from 1 (not at all) to 4 (very much), with higher scores indicating better functioning. The Social and Emotional Loneliness Scale for Adults (SELSA-S; DiTommaso et al., 2004) was used to examine social connectedness. The SELSA-S includes three subscales: Social $(\alpha=.90)$, Romantic $(\alpha=.87)$, and Family $(\alpha=.89)$. The 15 -item questionnaire uses a 7-point Likert scale ranging from 1 (strongly disagree) to 7 (strongly agree). A higher score on the SELSA-S represents high levels of emotional and social loneliness. The Adolescent and Adult Mindfulness Scale (AAMS; Droutman et al., 2018) was used to evaluating dispositional mindfulness. The AAMS includes four subscales: Attention and Awareness (AAMS: AA; $\alpha=.78$ ), Self Accepting (AAMS:SA; $\alpha=.77$ ), Non-Judgemental (AAMS:NonJ; $\alpha=.77$ ), and Non-Reactivity (AAMS; NonR; $\alpha=.77$ ). The 19-item questionnaire uses a 5-point Likert scale ranging from 1 (never true) to 5 (always true), with higher scores indicating increased mindfulness.

\section{Results}

Nolte et al. (2019) collected data from over 15,000 individuals (including 416 individuals who had a current cancer diagnosis) from Europe, Canada, and the United States to determine normative data for the QLQ-C30. The quality of life scores in the current study were similar to those reported by Nolte et al. (65.46 vs. 66.1; see Table 1) and slightly lower than the quality of life in a sample of individuals who were in remission or cured (Van Leeuwen et al., 2018).

Table 1. Mean/Standard Deviation on Relevant Variables as a Function of Time Since Diagnosis.

\begin{tabular}{lccccc}
\hline & Overall & $<2$ Years & $2-5$ Years & $5-10$ Years & $>10$ Years \\
\hline QLC-C30 Total & $65.46 / 18.44$ & $62.13 / 18.03$ & $64.54 / 19.00$ & $65.80 / 19.80$ & $67.61 / 19.09$ \\
Leisure engagement & $17.63 / 2.54$ & $17.88 / 2.84$ & $17.91 / 2.65$ & $16.89 / 2.11$ & $17.20 / 2.32$ \\
AAMS: AA & $3.20 / 0.88$ & $3.12 / 1 / 06$ & $3.22 / 0.87$ & $3.30 / 0 / 75$ & $3.32 / 0.91$ \\
AAMS: NR & $3.55 / 1.00$ & $3.35 / 0.96$ & $3.57 / 1.05$ & $3.65 / 0.84$ & $5.52 / 1.03$ \\
AAMS: NJ & $3.37 / 0.90$ & $3.17 / 0.94$ & $3.38 / 0.87$ & $3.27 / 0.73$ & $3.42 / 1.07$ \\
AAMS: SA & $3.57 / 0.98$ & $3.43 / 1.05$ & $3.53 / 0.97$ & $3.60 / 0.97$ & $3.69 / 1 / 11$ \\
SELSA: Family & $2.58 / 1.53$ & $2.37 / 1.46$ & $2.45 / 1 / 50$ & $2.54 / 1.58$ & $3.17 / 1 / 55$ \\
SELSA: Romantic & $3.43 / 1 / 82$ & $3.05 / 1.56$ & $3.39 / 1.87$ & $3.43 / 1.83$ & $3.83 / 1.94$ \\
SELSA: Social & $2.98 / 1.47$ & $2.70 / 1.32$ & $2.96 / 1.49$ & $2.96 / 1.52$ & $3.29 / 1.49$ \\
Medical support & $2.59 / 0.83$ & $2.71 / 0.69$ & $2.63 / 0.85$ & $2.59 / 0.77$ & $2.43 / 0.92$ \\
Age & $53.54 / 12.93$ & & & & \\
\hline
\end{tabular}


Further, in the current study, quality of life of participants did not improve in the years after their initial cancer diagnosis, $F(3,184)=0.39, p=.76$. Time since diagnosis did not affect overall social connectedness or leisure engagement.

A correlational analysis indicated that the correlations between the QLQ-C30 summary score and leisure engagement, medical support, and three mindfulness subscales of the AAMS (Self Accepting, Non-Judgemental, and Non-Reactivity) were statistically significant (see Table 2). Further, there were statistically significant correlations between AAMS: Non-Reacting, AAMS: Non-Judging, and AAMS: Self-Acceptance and QLQ:C30 Physical, Emotional, Cognitive, and Social Functioning scores. Higher leisure engagement was related to lower levels of loneliness and related specifically to the Self Acceptance subscale of the AAMS. Conversely, QoL scores were significantly and inversely related to family, social, and romantic loneliness.

A hierarchical regression predicting the QLQ-C30 summary Score was conducted. The model was statistically significant $F(13,117)=6.64, p<.001$ and accounted for $42.4 \%$ of the variability in QoL. To predict overall QoL, age, sex, and disease variables (years since diagnosis, relapse) were entered in the first block, medical support and loneliness (family, social, romantic) were entered in the second block, and finally, mindfulness subscales and leisure engagement were entered in the final block. Block 1 was not statistically significant, indicating that demographic variables and disease characteristics did not contribute significantly to the overall model. Block 2 was statistically significant $\left(R^{2}\right.$ change $\left.=.199\right)$ with family loneliness $(p=<.001)$ and medical support $(p=.05)$ contributing significantly to the model. Block 3 was also significant $\left(R^{2}\right.$ change $\left.=.186\right)$ with AAMS: Self-Acceptance (mindfulness subscale; $\left.p=.02\right)$ and leisure engagement $(p=.01)$ contributing significantly to the model. Thus, individuals who report lower family loneliness as well as greater medical support, self-acceptance, and leisure engagement had higher global quality of life.

Table 2. Correlations between Quality of Life (QLQ-C30), Years since Diagnosis, Loneliness, and Leisure Engagement.

\begin{tabular}{|c|c|c|c|c|c|c|c|c|c|c|}
\hline & $\begin{array}{c}\text { QLQ- } \\
\text { C30 }\end{array}$ & Leisure & AAMSAA & $\begin{array}{l}\text { AAMS } \\
\text { NR }\end{array}$ & $\begin{array}{l}\text { AAMS } \\
\text { NJ }\end{array}$ & $\begin{array}{c}\text { AAMS } \\
\text { SA }\end{array}$ & $\begin{array}{l}\text { SELSA } \\
\text { Family }\end{array}$ & $\begin{array}{c}\text { SELSA } \\
\text { Romantic }\end{array}$ & $\begin{array}{c}\text { SELSA } \\
\text { Social }\end{array}$ & $\begin{array}{l}\text { Medical } \\
\text { support }\end{array}$ \\
\hline $\begin{array}{l}\text { QLQ-C30 } \\
\text { Leisure } \\
\text { engagement }\end{array}$ & $-.361 * *$ & & & & & & & & & \\
\hline AAMS-AA & .001 & $.217 * *$ & & & & & & & & \\
\hline AAMS-NR & $.363 * *$ & -.004 & -.060 & & & & & & & \\
\hline AAMS-NJ & $.262 * *$ & .098 & $-.506^{* *}$ & $.410 * *$ & & & & & & \\
\hline AAMS-SA & $.476^{* *}$ & $.189 *$ & -.065 & $.585 * *$ & $.547 * *$ & & & & & \\
\hline SELSA-F & $-.354 * *$ & $-.396 * *$ & -.040 & $-.238 * *$ & -.051 & $-.289 * *$ & & & & \\
\hline SELSA-R & $-.187 * *$ & $-.360 * *$ & -.065 & -.119 & -.023 & $-.162 *$ & $.554 * *$ & & & \\
\hline SELSA-S & $-.240 * *$ & $-.484 * *$ & -.131 & $-.226 * *$ & -.006 & $-.268 * *$ & $.581 * *$ & $.403 * *$ & & \\
\hline $\begin{array}{l}\text { Medical } \\
\text { support }\end{array}$ & $.259 * *$ & $.251 * *$ & .013 & $.153^{*}$ & -.011 & $.150^{*}$ & $-.370 * *$ & $-.204 * *$ & $-.335 * *$ & \\
\hline $\begin{array}{l}\text { Years Since } \\
\text { Diagnosis }\end{array}$ & -.037 & -.102 & .059 & -.101 & -.031 & -.020 & $.21 * *$ & .078 & .082 & -.096 \\
\hline
\end{tabular}

\section{Discussion}

As the result of medical advances and novel treatment methods, many individuals will survive a cancer diagnosis and, at some point after treatment, return to everyday activities. Researchers have traditionally focused mainly on the impact that cancer has on physical and psychological health during diagnosis and treatment. Thus, the goal for many patients and their medical team has been to get through treatment with the goal of returning to a "normal" life, with "normal" activities. Although some psychological and emotional consequences of diagnosis and treatment are not as immediately significant, their importance for survivors increases in the months and years after a cancer diagnosis. Although QoL measures are created to assess global functioning and include scales to assess both current symptoms and overall physical, emotional, and cognitive functioning, other lifestyle factors influence physical health beyond the characteristics related specifically to cancer and its diagnosis. Thus, our goal was to examine how self-reported social support (family and medical) and leisure engagement affected overall wellness in a sample of cancer survivors.

In this study, disease characteristics, including the time that has passed since diagnosis and cancer relapse, did not significantly contribute to overall quality of life. Further, QLQ:C30 scores of current participants were significantly lower than scores of cancer survivors (Van Leeuwen et al., 2018), likely 
because some of the current participants were newly diagnosed and not yet through their treatment. Although physical symptoms and functioning might improve in the years after treatment, education and programs focused on social and emotional functioning could improve overall quality of life. This research also highlights that the impact of medical and family support extends beyond disease characteristics in cancer survivors. Cancer survivors who have a strong support network, that includes their family and medical care team, and a higher level of autonomy in their treatment process report better physical and mental health functioning.

At the correlational level, increased mindfulness (apart from acting with awareness) was associated with higher quality of life. Further, in regression analyses, the self-acceptance component of mindfulness predicted overall quality of life, which lends insight into how different aspects of mindfulness can influence a patient's QoL. These results are in line with Best et. al (2019) who reported that aspects of mindfulness that focus on an awareness of bodily experiences might increase positive and negative physical experiences. In the current study, self acceptance, a mindfulness subscale that focuses on acceptance of personal emotions predicted higher QoL. Individuals that label their emotions "wrong" or think that they "shouldn't be feeling this way" have low levels of self-acceptance and lower quality of life. The current results replicate Garland et al. (2017) and indicate that being able to accept your own emotions, even if they are negative, was related to more positive outcomes.

Previous studies have examined the influence of leisure satisfaction by specifically examining how much an individual is enjoying their leisure activity as a predictor of health; however, this study examines how a variety of leisure activities impacts overall QoL. This is an important distinction; leisure engagement is not activity dependent but focuses on whether individuals "switch it up" and experience a variety of solitary and social activities. In this case, the more the better. Chun and colleagues (2016) found that increased leisure satisfaction in cancer survivors improved their sense of purpose in life and the current results extends these results. Further, the current results replicated research that has shown the positive impacts of leisure activities and mindfulness on overall QoL in cancer patients (Courtens et al., 1996; Fangel et al., 2013; Garland et al., 2017), but takes it a step further and examines what specific aspects of these variables are have the most impact.

Loneliness has been shown to negatively impact cancer outcomes (Garssen, 2004; Kroenke et al., 2006). We examined three types of loneliness (Family, Social, Romantic). All three components of loneliness were significantly correlated the overall QoL scores; however, only Family loneliness was a significant predictor in the current model. It is possible that given the age of the sample $\left(\mathrm{M}_{\mathrm{age}}=53.54\right)$ and the fact that $62.5 \%$ were currently in a romantic relationship, the measurement of "family" may include one's romantic partner. Previous research further supports that higher family support in patients with chronic illness improves medical compliance, which may improve overall health (Mongan, 2017).

Medical support was also a statistically significant predictor of quality of life, indicating that having a positive relationship with healthcare providers has a positive impact. Medical support questions included ratings on how happy participants were with their involvement in their treatment choices and how much they felt listened to in the process. It is possible that when patients feel comfortable with their practitioners and understand their medical treatment, their treatment compliance and satisfaction with disease outcomes increases. Current literature supports that a positive patient-clinician relationship improves medical compliance in cancer patients (Chou et al., 2017).

\section{Conclusion}

This study further examined the relationship between QoL in cancer survivors and focused on modifiable lifestyle variables that could improve quality of life and functioning. Factors such as medical and family support, acceptance of one's positive and negative feelings and integration of a variety of leisure activities into their life are significantly associated with higher levels of QoL. These findings are useful in the hands of health care practitioners who are interacting with patients during the course of cancer diagnosis and treatment. Encouraging family support, including the patient in the decision-making process, encouraging a variety of physically possible leisure activities and normalizing negative emotions surrounding diagnosis and disease symptoms are all ways that overall QoL can be improved.

\section{References}

Aaronson, N. K., Ahmedzai, S., Bergman, B., Bullinger, M., Cull, A., Duez, N. J., Filiberti, A., Flechtner, H., Fleishman, S. B., \& de Haes, J. C. (1993). The European Organization for Research and Treatment of Cancer QLQ-C30: A quality-of-life instrument for use in international clinical trials in oncology. Journal of the National Cancer Institute, 85(5), 365-376. 
Beard, J. G., \& Ragheb, M. G. (1980). Measuring leisure satisfaction. Journal of Leisure Research, 12(1), 20-33.

Best, L., Proctor, C., Freeze, T., Gaudet, D., Russel, R., McPhee., R (2019). Relation between subjective and physical well-being and mindfulness. Oral presentation at the InPact 2017 International Psychological Applications Conference and Trends. Zagreb, Croatia.

Chou, P.-L., Rau, K.-M., Yu, T.-W., H., T-L., Sun, J.-L., Wang, S.-Y., \& Lin, C.-C. (2017). Patient-clinician relationship seems to affect adherence to analgesic use in cancer patients: A cross sectional study in a Taiwanese population. International Journal for Quality in Health Care, 29(7), 935-940.

Chun, S., Heo, J., Lee, S., \& Kim, J. (2016). Leisure-related predictors on a sense of purpose in life among older adults with cancer. Activities, Adaptation \& Aging, 40(3), 266-280.

Courtens, A. M., Stevens, F. C. J., Crebolder, H. F. J. M., \& Philipsen, H. (1996). Longitudinal study on quality of life and social support in cancer patients. Cancer Nursing, 19(3), 162-169.

Deckx, L., van den Akker, M., \& Buntinx, F. (2014). Risk factors for loneliness in patients with cancer: A systematic literature review and meta-analysis. European Journal of Oncology Nursing, 18(5), 466-477.

DiTommaso, E., Brannen, C., \& Best, L. A. (2004). Social and Emotional Loneliness Scale for Adults-Short Version [Database record]. PsycTESTS.

Drennan, J., Treacy, M., Butler, M., Byrne, A., Fealy, F., Frazer, K., \& Irving, K. (2008). The experience of social and emotional loneliness among older people in Ireland. Ageing and Society, 28(8), 113-1132.

Droutman, V., Golub, Ilana, Oganesyan, A., \& Read, S. (2018). Development and initial validation of the Adolescent and Mindfulness Scale (AAMS). Personality and Individual Differences, 123, 34-43.

Fangel, L. M. V., Panobianco, M. S., Kebbe, L. M., de Almeida, A. M., \& de Oliveira Gozzo T. (2013). Quality of life and daily activities performance after breast cancer treatment. Acta Paulista de Enfermagem, 26(1), 93-100.

Garland, E. L., Thielking, P., Thomas, E. A., Coombs, M., White, S., Lombardi, J., \& Beck, A. (2017). Linking dispositional mindfulness and positive psychological processes in cancer survivorship: A multivariate path analytic test of the mindfulness-to-meaning theory. Psycho-oncology, 26(5), 686-692.

Garssen, B. (2004). Psychological factors and cancer development: Evidence after 30 years of research. Clinical Psychology Review, 24(3), 315-338.

Hays, R. D., \& Morales, L. S. (2001). The RAND-36 measure of health-related quality of life. Annals of Medicine, 33(5), 350-357.

Kroenke, C. H., Kubzansky, L. D., Schernhammer, E. S., Holmes, M. D., \& Kawachi, I. (2006). Social networks, social support, and survival after breast cancer diagnosis. Journal of Clinical Oncology, 24(7), 1105-1111.

Langeveld, N. E., Grootenhuis, M. A., Voute, P. A., De Haan, R. J., \& Van Den Bos, C. (2004). Quality of life, self-esteem, and worries in young adult survivors of childhood cancer. Psycho-Oncology, 13(12), 867-881.

Liu, X., Li, J., Zhang, Q., Zhao, Y., \& Xu, W. (2021). Being beneficial to self and caregiver: The role of dispositional mindfulness among breast cancer patients. Supportive Care in Cancer, 29(1), 239-246.

Mongan, R., \& Fajar, F. (2017). Relationship between family support and medical compliance in patients with pulmonary tuberculosis in the working area of the community health center of Abeli, Kendari. Public Health of Indonesia, 3(1), 17-22.

Nayak, M. G., George, A., Vidyasagar, M. S., Mathew, S., Nayak, S., Nayak, B. S., Shashidhara, Y. N., \& Kamath, A. (2017). Quality of life among cancer patients. Indian Journal of Palliative Care, 23(4), 445-450.

Nolte, S., Liegl, G., Petersen, M. A., Aaronson, N. K., Costantini, A., Fayers, P. M., ... \& EORTC Quality of Life Group. (2019). General population normative data for the EORTC QLQ-C30 health-related quality of life questionnaire based on 15,386 persons across 13 European countries, Canada and the Unites States. European journal of cancer, 107, 153-163.

Van Leeuwen, M., Husson, O., Alberti, P., Arraras, J. I., Chinot, O. L., Costantini, A., ... \& van de Poll-Franse, L. V. (2018). Understanding the quality of life (QOL) issues in survivors of cancer: towards the development of an EORTC QOL cancer survivorship questionnaire. Health and Quality of life Outcomes, 16(1), 1-15.

Shipp, S., McKinstry, C., \& Pearson, E. (2015). The impact of colorectal cancer on leisure participation: A narrative study. British Journal of Occupational Therapy, 78(5), 311-319.

World Health Organization. (2021, March 3). Cancer. Retrieved March 20, 2021, from https://www.who.int/news-room/fact-sheets/detail/cancer 